\title{
Improvement in Seed Yield and Related Traits of Linseed Genotypes (Linum usitatissimum L.) through Various Selection Parameters in Mid-Hills of North-West Himalayas
}

\author{
Satish Paul, Nimit Kumar* and Pankaj Chopra
}

\begin{abstract}
Department of Crop Improvement, CSK Himachal Pradesh Krishi Vishvavidyalaya, Palampur-176062, India

*Corresponding author
\end{abstract}

\section{A B S T R A C T}

Keywords

Linum

usitatissimum L., Correlation, Path analysis, Principal component analysis.

Article Info

Accepted:

24 January 2017

Available Online:

10 February 2017
Forty five linseed genotypes were subjected to study the interrelationship among the traits at the Experimental Farm of the Department of Crop Improvement, CSK Himachal Pradesh Krishi Vishvavidyalaya, Palampur, during rabi 2015-2016. The genotypic and phenotypic correlation coefficient obtained between different traits was similar in direction, while in magnitude, genotypic correlation was higher than the corresponding phenotypic correlations for most of the traits. Seed yield per plant had maximum significant and positive genotypic and phenotypic association with biological yield per plant followed by harvest index and seeds per capsule. Harvest index plant contributed indirectly through plant height followed by technical height and biological yield per plant has maximum indirect effects through capsules per plant on seed yield per plant. Principal component analysis (PCA) showed that seed yield per plant, harvest index, biological yield per plant seeds per capsule and capsules per plant were found in same group.

\section{Introduction}

Lineed (Linum usitatissimum L.; $\mathrm{n}=15$ ) is an important oilseed crop which is the only species in Linaceae family with economic values (Tadesse et al., 2009). It has nutrients and pharmaceutical uses and used for edible and lightening purposes and also in animal fat and poultry diets (Khan et al., 2010). Linseed contain $30-45 \%$ oil, making it an important industrial crop. It has high unsaturated fatty acids, especially Linolenic acid (Khan et al., 2010). Due to less impression of direct selection for yield, more efforts should be over indirect selection for yield components. Proper understanding of association of different traits, provide more reliable selection criterion to achieve a high seed yield (Akbar et al., 2001). Correlation coefficients may not evolve satisfactory results in uncovering the real interrelationships among the traits. Nevertheless, selection for yield via highly correlated traits becomes easy if the contribution of different characters to yield is quantified using path coefficient analysis (Dewey \& Lu, 1959). Multivariate statistical techniques which simultaneously analyze multiple measurements on each individual under investigation are widely used in analysis of interrelationships. Among the 
multivariate techniques, principal component analysis (PCA) had been shown to be very useful in selecting genotypes for breeding program that meet the objective of a plant breeder (Mohammadi and Prasanna, 2003). PCA may be used to reveal patterns and eliminate redundancy in data sets (Adams, 1995) as morphological and physiological variations routinely occur in crop species. The present investigation was carried out to study the associations among yield and yield related traits in linseed.

\section{Materials and Methods}

Interrelationships for various traits was studied in 45 local collection (Table 1) of linseed during rabi 2015-16 at Experimental Farm of the Department of Crop Improvement, CSK Himachal Pradesh Krishi Vishvavidyalaya, Palampur, India $\left(32^{\circ} 8^{\prime} \mathrm{N}\right.$, $76^{\circ} 3^{\prime}$ E) represents humid sub-temperate climate zone with annual rainfall of $2500 \mathrm{~mm}$ and acidic soil with $\mathrm{pH}$ of 5.0 to 5.6. The experiment was conducted using randomized complete block design with three replications. Each replication consisted of three rows of each genotype. Row to row distance was 30 $\mathrm{cm}$ with row length of 3 meter and plant to plant distance was $10 \mathrm{~cm}$. Data was recorded on five randomly selected plants in each replication for plant height, technical height, primary branches per plant, secondary branches per plant, capsules per plant, biological yield per plant, seeds per capsule, seed yield per plant and harvest index calculated as:-

Harvest index $(\%)=\frac{\text { Seed yield per plant }}{\text { Biological yield per plant }} \times 100$

Statistical analysis: The recorded data was subjected to analysis of genotypic and phenotypic correlation coefficients as suggested by Al- Jibouri et al., (1958) and the path coefficient analysis was conducted as suggested by Dewey and Lu (1959).
PCA: Principal component analysis (PCA) analysis was performed using XLSTAT software to determine the best relationships among characters.

\section{Results and Discussion}

The possible increase the seed yield through yield related traits, as primary target of crop improvement, requires understanding the inter relationship between various yield contributing traits or in fact, yield components. The correlation coefficients between different characters are given in Table 2. The results of experiment revealed that the traits plant height, secondary branches per plant, capsules per plant, biological yield per plant, seeds per capsule and harvest index had the strong positive association with seed yield per plant at both genotypic and phenotypic levels. Whereas, technical height and primary branches per plant exhibited positive but non-significant associations with seed yield per plant. The genotypic and phenotypic correlation coefficient was similar in directions, while in magnitude, genotypic correlations were mostly higher than corresponding phenotypic correlations. Similar findings were reported by Sohan et al., (2004), Joshi (2004). Thus the low phenotypic correlation could results due to the masking and modifying effect of environment on the association of characters at genotypic level. On the basis of present studies, it can be concluded that the selection based on traits viz., plant height, secondary branches per plant, capsules per plant, biological yield per plant, seeds per capsule and harvest can provide better result for improvement of seed yield per plant in linseed, as earlier reported by Tariq et al., (2014) plant height, number of capsules per plant, number of seeds per capsule, Yadav (2001) for number of capsules per plant and number of seeds per capsule, Muhammad et al., (2003) for number of branches per plant. 
Table.1 Details of material used in present study

\begin{tabular}{|c|c|c|c|c|c|c|}
\hline \multirow{2}{*}{$\begin{array}{l}\text { S. } \\
\text { No. }\end{array}$} & \multirow{2}{*}{$\begin{array}{l}\text { Collection } \\
\text { No. }\end{array}$} & \multirow{2}{*}{$\begin{array}{l}\text { Crop } \\
\text { Name }\end{array}$} & \multirow[t]{2}{*}{ Botanical Name } & \multicolumn{2}{|c|}{ Site of collection } & \multirow[t]{2}{*}{ State } \\
\hline & & & & Village & Disst. & \\
\hline 1 & KLSA-1 & Linseed & Linum usitatissimum & Utrala & Kangra & H.P. \\
\hline 2 & KLSA-2 & Linseed & Linum usitatissimum & Harer Baijnath & Kangra & H.P. \\
\hline 3 & KLSA-3 & Linseed & Linum usitatissimum & Drugh Nala Baijnath & - & H.P. \\
\hline 4 & KLSA-4 & Linseed & Linum usitatissimum & Harer Baijnath & Kangra & H.P. \\
\hline 5 & KLSA-5 & Linseed & Linum usitatissimum & Balh Harer Baijnath & Kangra & H.P. \\
\hline 6 & KLSA-6 & Linseed & Linum usitatissimum & Balh Harer Baijnath & Kangra & H.P. \\
\hline 7 & KLSA-7 & Linseed & Linum usitatissimum & Dramlu Harer Baijnath & Kangra & H.P. \\
\hline 8 & KLSA-8 & Linseed & Linum usitatissimum & Dramlu Harer Baijnath & Kangra & H.P. \\
\hline 9 & KLSA-9 & Linseed & Linum usitatissimum & Kholi Deol Baijnath & Kangra & H.P. \\
\hline 10 & KLSA-10 & Linseed & Linum usitatissimum & Phatar & Kangra & H.P. \\
\hline 11 & KLSA-11 & Linseed & Linum usitatissimum & Bhattu & - & H.P. \\
\hline 12 & KLSA-12 & Linseed & Linum usitatissimum & Tramal & - & H.P. \\
\hline 13 & KLSA-13 & Linseed & Linum usitatissimum & Dak Bangra Chauntra & Mandi & H.P. \\
\hline 14 & KLSA-14 & Linseed & Linum usitatissimum & Chauntra Joginder Nagar & Mandi & H.P. \\
\hline 15 & KLSA-15 & Linseed & Linum usitatissimum & Hara Bagh Joginder Nagar & Mandi & H.P. \\
\hline 16 & KLSA-16 & Linseed & Linum usitatissimum & Hara Bagh Joginder Nagar & Mandi & H.P. \\
\hline 17 & KLSB-1 & Linseed & Linum usitatissimum & Jia & Kangra & H.P. \\
\hline 18 & KLSB-2 & Linseed & Linum usitatissimum & Jia & Kangra & H.P. \\
\hline 19 & KLSB-3 & Linseed & Linum usitatissimum & Jia & Kangra & H.P. \\
\hline 20 & KLSB-4 & Linseed & Linum usitatissimum & Jagehar & Kangra & H.P. \\
\hline 21 & KLSB-5 & Linseed & Linum usitatissimum & Chamotu Jia & Kangra & H.P. \\
\hline 22 & KLSB-6 & Linseed & Linum usitatissimum & Chamotu Jia & Kangra & H.P. \\
\hline 23 & KLSB-7 & Linseed & Linum usitatissimum & Chamotu Jia & Kangra & H.P. \\
\hline 24 & KLC-3 & Linseed & Linum usitatissimum & Nagehar & - & H.P. \\
\hline 25 & KLC-4 & Linseed & Linum usitatissimum & Nagehar & - & H.P. \\
\hline 26 & KLC-9 & Linseed & Linum usitatissimum & Baijnath & Kangra & H.P. \\
\hline 27 & KLC-10 & Linseed & Linum usitatissimum & Arki & Solan & H.P. \\
\hline 28 & KLC-11 & Linseed & Linum usitatissimum & Ahju Baijnath & Kangra & H.P. \\
\hline 29 & KLC-12 & Linseed & Linum usitatissimum & Kandi ,Palampur & Kangra & H.P. \\
\hline 30 & KLC-13 & Linseed & Linum usitatissimum & Trehal ,Baijnath & Kangra & H.P. \\
\hline 31 & KLC-14 & Linseed & Linum usitatissimum & Utrala Baijnath & Kangra & H.P. \\
\hline 32 & KLD-1 & Linseed & Linum usitatissimum & Patti Panchrukhi & Kangra & H.P. \\
\hline 33 & KLD-2 & Linseed & Linum usitatissimum & Patti Panchrukhi & Kangra & H.P. \\
\hline 34 & KLD-3 & Linseed & Linum usitatissimum & Chandropa Patti Panchrukhi & Kangra & H.P. \\
\hline 35 & KLD-4 & Linseed & Linum usitatissimum & Palah Patti Panchrukhi & Kangra & H.P. \\
\hline 36 & KLD-5 & Linseed & Linum usitatissimum & Patti Panchrukhi & Kangra & H.P. \\
\hline 37 & KLD-6 & Linseed & Linum usitatissimum & Chandropa Patti Panchrukhi & Kangra & H.P. \\
\hline 38 & KLD-7 & Linseed & Linum usitatissimum & Palah Patti Panchrukhi & Kangra & H.P. \\
\hline 39 & KLD-8 & Linseed & Linum usitatissimum & Palah Patti Panchrukhi & Kangra & H.P. \\
\hline 40 & KLD-10 & Linseed & Linum usitatissimum & Chandropa Patti Panchrukhi & Kangra & H.P. \\
\hline 41 & T-397 & Linseed & Linum usitatissimum & & & \\
\hline 42 & Baner & Linseed & Linum usitatissimum & & & \\
\hline 43 & Nagarkot & Linseed & Linum usitatissimum & & & \\
\hline 44 & Him Alsi-2 & Linseed & Linum usitatissimum & & & \\
\hline 45 & Himani & Linseed & Linum usitatissimum & & & \\
\hline
\end{tabular}


Table.2 Estimates of genotypic $(\mathrm{G})$ and phenotypic $(\mathrm{P})$ correlation coefficients among different traits of linseed

\begin{tabular}{|c|c|c|c|c|c|c|c|c|c|}
\hline & & $\begin{array}{l}\text { Technical } \\
\text { height } \\
(\mathrm{cm})\end{array}$ & $\begin{array}{l}\text { Primary } \\
\text { branches } \\
\text { per plant }\end{array}$ & $\begin{array}{l}\text { Secondary } \\
\text { branches } \\
\text { per plant }\end{array}$ & $\begin{array}{c}\text { Capsules } \\
\text { per } \\
\text { plant }\end{array}$ & $\begin{array}{l}\text { Biological } \\
\text { yield per } \\
\text { plant }(\mathrm{gm})\end{array}$ & $\begin{array}{l}\text { Seeds per } \\
\text { capsule }\end{array}$ & $\begin{array}{c}\text { Harvest } \\
\text { index } \\
(\%)\end{array}$ & $\begin{array}{l}\text { Seed yield } \\
\text { per plant } \\
(\mathrm{gm})\end{array}$ \\
\hline \multirow{2}{*}{ Plant Height (cm) } & $\mathrm{P}$ & $.4987^{* *}$ & $.1835^{*}$ & $.2110^{*}$ & .0880 & .0502 & .0907 & $.2134^{*}$ & $.1682^{*}$ \\
\hline & G & $.6970^{* *}$ & $.2043^{*}$ & $.2035^{*}$ & .1138 & .0560 & $.2237^{* *}$ & $.2862^{* *}$ & $.1686^{*}$ \\
\hline \multirow{2}{*}{ Technical height $(\mathrm{cm})$} & $\mathrm{P}$ & & .0571 & .0749 & -.0954 & -.0981 & $.1687^{*}$ & $.1948^{*}$ & .0292 \\
\hline & G & & .0765 & .1092 & -.1084 & -.1229 & $.3203^{* *}$ & $.2315^{* *}$ & .0208 \\
\hline \multirow{2}{*}{ Primary branches per plant } & $\mathrm{P}$ & & & $.8811^{* *}$ & $.6562^{* *}$ & .0931 & $.3201^{* *}$ & -.0200 & .1042 \\
\hline & $\mathrm{G}$ & & & $.9741^{* *}$ & $.7206^{* *}$ & .1031 & $.6052^{* *}$ & -.0340 & .0986 \\
\hline \multirow{2}{*}{$\begin{array}{l}\text { Secondary branches per } \\
\text { plant }\end{array}$} & $P$ & & & & $.6153^{* *}$ & .1257 & $.2676^{* *}$ & .0090 & $.1715^{*}$ \\
\hline & $\mathrm{G}$ & & & & $.6978^{* *}$ & .1410 & $.6234^{* *}$ & .0141 & $.1941^{*}$ \\
\hline \multirow{2}{*}{ Capsules per plant } & $\mathrm{P}$ & & & & & $.2089^{*}$ & $.1700^{*}$ & -.0802 & $.1827^{*}$ \\
\hline & $\mathrm{G}$ & & & & & $.2117^{*}$ & $.2544^{* *}$ & -.0995 & $.1825^{*}$ \\
\hline \multirow{2}{*}{$\begin{array}{l}\text { Biological yield per plant } \\
\text { (gm) }\end{array}$} & $\mathrm{P}$ & & & & & & -.0943 & $-.3133^{* *}$ & $.5534^{* *}$ \\
\hline & $\mathrm{G}$ & & & & & & -.1790 & $-.3263^{* *}$ & $.5701^{* *}$ \\
\hline \multirow{2}{*}{ Seeds per capsule } & $\mathrm{P}$ & & & & & & & -.0455 & $.2051^{*}$ \\
\hline & G & & & & & & & -.1233 & $.2180^{*}$ \\
\hline \multirow{2}{*}{ Harvest index (\%) } & $\mathrm{P}$ & & & & & & & & $.5180^{* * *}$ \\
\hline & $\mathrm{G}$ & & & & & & & & $.5377^{* *}$ \\
\hline
\end{tabular}

Table.4 Eigenvectors and eigen values of 4 principal components for 9 characters of 45 linseed genotypes

\begin{tabular}{|c|c|c|c|c|c|c|c|c|c|c|c|c|}
\hline & $\begin{array}{c}\text { Plant } \\
\text { Height } \\
\text { (cm) }\end{array}$ & $\begin{array}{c}\text { Technical } \\
\text { height } \\
\text { (cm) }\end{array}$ & $\begin{array}{c}\text { Primary } \\
\text { branches } \\
\text { per } \\
\text { plant }\end{array}$ & $\begin{array}{c}\text { Secondary } \\
\text { branches } \\
\text { per plant }\end{array}$ & $\begin{array}{c}\text { Capsules } \\
\text { per } \\
\text { plant }\end{array}$ & $\begin{array}{l}\text { Biological } \\
\text { yield per } \\
\text { plant } \\
(\text { gm) }\end{array}$ & $\begin{array}{c}\text { Seeds } \\
\text { per } \\
\text { capsule }\end{array}$ & $\begin{array}{c}\text { Harvest } \\
\text { index } \\
(\%)\end{array}$ & $\begin{array}{l}\text { Seed } \\
\text { yield } \\
\text { per } \\
\text { plant } \\
(\text { gm) }\end{array}$ & Eigenvalue & $\begin{array}{c}\text { Variability } \\
(\%)\end{array}$ & $\begin{array}{c}\text { Cumulative } \\
(\%)\end{array}$ \\
\hline PC1 & 0.230 & 0.110 & 0.524 & 0.529 & 0.453 & 0.200 & 0.256 & 0.109 & 0.242 & 2.956 & 32.844 & 32.844 \\
\hline PC2 & 0.005 & -0.163 & -0.185 & -0.147 & 0.010 & 0.527 & -0.399 & 0.328 & 0.610 & 2.067 & 22.963 & 55.807 \\
\hline PC3 & 0.615 & 0.640 & -0.147 & -0.118 & -0.231 & -0.113 & 0.034 & 0.329 & 0.040 & 1.674 & 18.600 & 74.407 \\
\hline PC4 & -0.064 & -0.229 & 0.080 & 0.082 & 0.243 & -0.533 & -0.342 & 0.670 & -0.144 & 0.978 & 10.866 & 85.273 \\
\hline
\end{tabular}


Table.3 Estimates of genotypic $(\mathrm{G})$ and phenotypic $(\mathrm{P})$ direct and indirect effects of different traits on seed yield in linseed

\begin{tabular}{|c|c|c|c|c|c|c|c|c|c|c|}
\hline & & $\begin{array}{c}\text { Plant } \\
\text { Height } \\
\text { (cm) }\end{array}$ & $\begin{array}{c}\text { Technical } \\
\text { height } \\
(\mathrm{cm})\end{array}$ & $\begin{array}{c}\text { Primary } \\
\text { branches } \\
\text { per } \\
\text { plant }\end{array}$ & $\begin{array}{c}\text { Secondary } \\
\text { branches } \\
\text { per plant }\end{array}$ & $\begin{array}{c}\text { Capsules } \\
\text { per } \\
\text { plant }\end{array}$ & $\begin{array}{c}\text { Biological } \\
\text { yield per } \\
\text { plant } \\
(\text { gm) }\end{array}$ & $\begin{array}{c}\text { Seeds } \\
\text { per } \\
\text { capsule }\end{array}$ & $\begin{array}{c}\text { Harvest } \\
\text { index } \\
(\%)\end{array}$ & $\begin{array}{c}\text { Seed } \\
\text { yield } \\
\text { per } \\
\text { plant } \\
(\mathrm{gm}) \\
\end{array}$ \\
\hline \multirow{2}{*}{ Plant Height (cm) } & $\mathrm{P}$ & -.1041 & .0004 & -.0182 & .0244 & .0072 & .0398 & .0451 & .1736 & $.1682^{*}$ \\
\hline & $\mathrm{G}$ & -.1320 & .0131 & -.1173 & .1113 & .0157 & .0447 & .0041 & .2290 & $.1686^{*}$ \\
\hline \multirow{2}{*}{ Technical height (cm) } & $\mathrm{P}$ & -.0519 & .0009 & -.0057 & .0087 & -.0079 & -.0779 & .0046 & .1584 & .0292 \\
\hline & $\mathrm{G}$ & -.0920 & .0188 & -.0439 & .0597 & -.0149 & -.0980 & .0058 & .1852 & .0208 \\
\hline \multirow{2}{*}{ Primary branches per plant } & $\mathrm{P}$ & -.0191 & .0000 & -.0994 & .1020 & .0541 & .0739 & .0089 & -.0162 & .1042 \\
\hline & $\mathrm{G}$ & -.0270 & .0014 & -.5738 & .5328 & .0992 & .0822 & .0110 & -.0272 & .0986 \\
\hline \multirow{2}{*}{ Secondary branches per plant } & $\mathrm{P}$ & -.0220 & .0001 & -.0876 & .1158 & .0507 & .0998 & .0075 & .0073 & $.1715^{*}$ \\
\hline & G & -.0269 & .0021 & -.5590 & .5470 & .0961 & .1124 & .0113 & .0113 & $.1941^{*}$ \\
\hline \multirow{2}{*}{ Capsules per plant } & $\mathrm{P}$ & -.0092 & -.0001 & -.0652 & .0713 & .0824 & .1658 & .0031 & -.0653 & $.1827^{*}$ \\
\hline & $\mathrm{G}$ & -.0150 & -.0020 & -.4135 & .3817 & .1376 & .1687 & .0046 & -.0796 & $.1825^{*}$ \\
\hline \multirow{2}{*}{ Biological yield per plant (gm) } & $\mathrm{P}$ & -.0052 & -.0001 & -.0093 & .0146 & .0172 & .7937 & -.0026 & -.2548 & $.5534^{* *}$ \\
\hline & $\mathrm{G}$ & -.0074 & -.0023 & -.0592 & .0771 & .0291 & .7971 & -.0032 & -.2611 & $.5701^{* *}$ \\
\hline \multirow{2}{*}{ Seeds per capsule } & $\mathrm{P}$ & -.0094 & .0001 & -.0318 & .0310 & .0091 & -.0749 & .3180 & -.0370 & $.2051^{*}$ \\
\hline & $\mathrm{G}$ & -.0295 & .0060 & -.3473 & .3409 & .0350 & -.1427 & .4542 & -.0986 & $.2180^{*}$ \\
\hline \multirow{2}{*}{ Harvest index (\%) } & $\mathrm{P}$ & -.0222 & .0002 & .0020 & .0010 & -.0066 & -.2487 & -.0013 & .8002 & $.5180^{* *}$ \\
\hline & $\mathrm{G}$ & -.0378 & .0043 & .0195 & .0077 & -.0137 & -.2601 & -.0022 & .8133 & $.5377^{\text {** }}$ \\
\hline
\end{tabular}


Fig.1 Scree plot of 45 genotypes of linseed on principal components 1-9 (F1-F9)

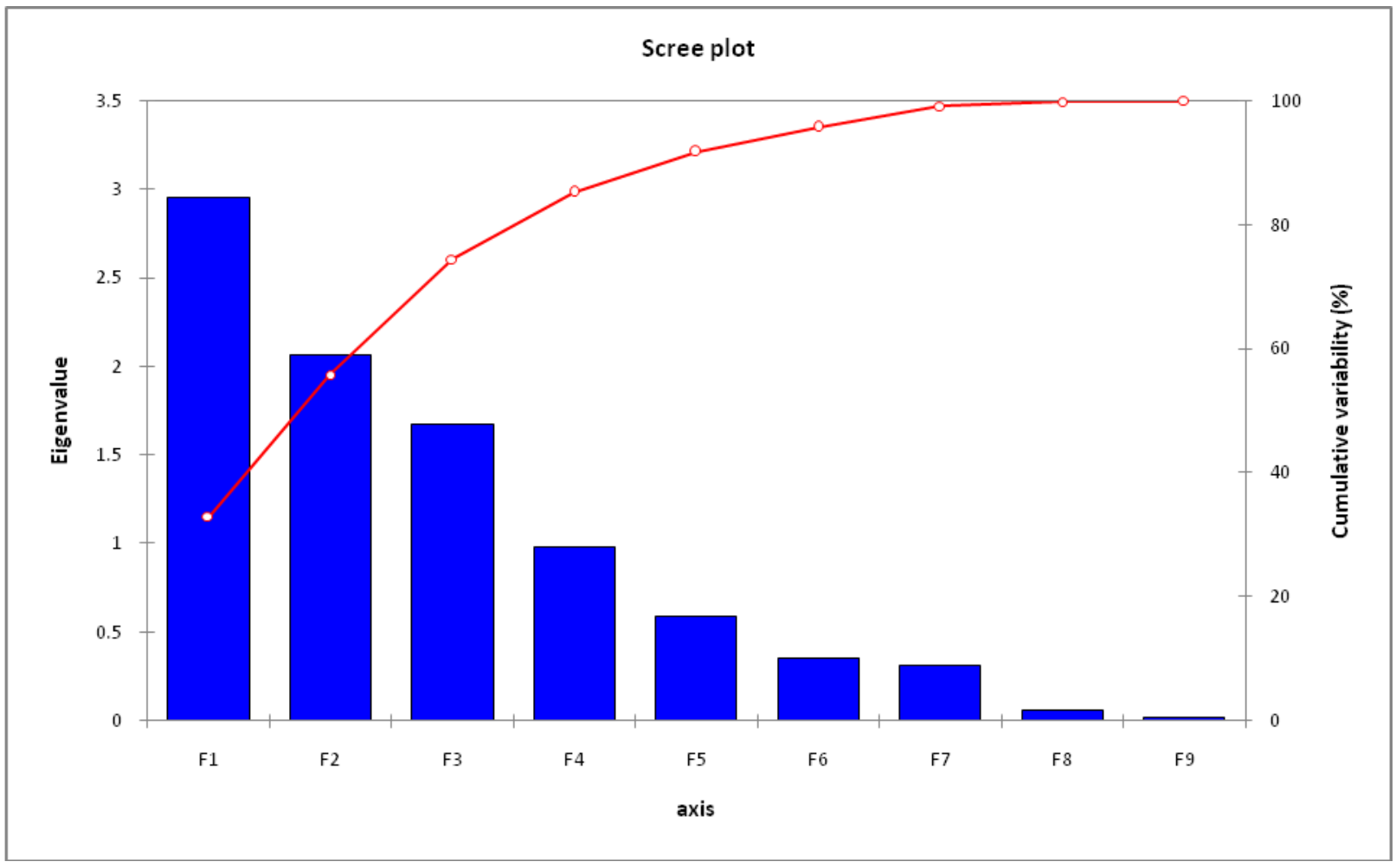

Fig.2 Biplot of 45 genotypes of linseed on Principal Component (F1 and F2) axis I and II

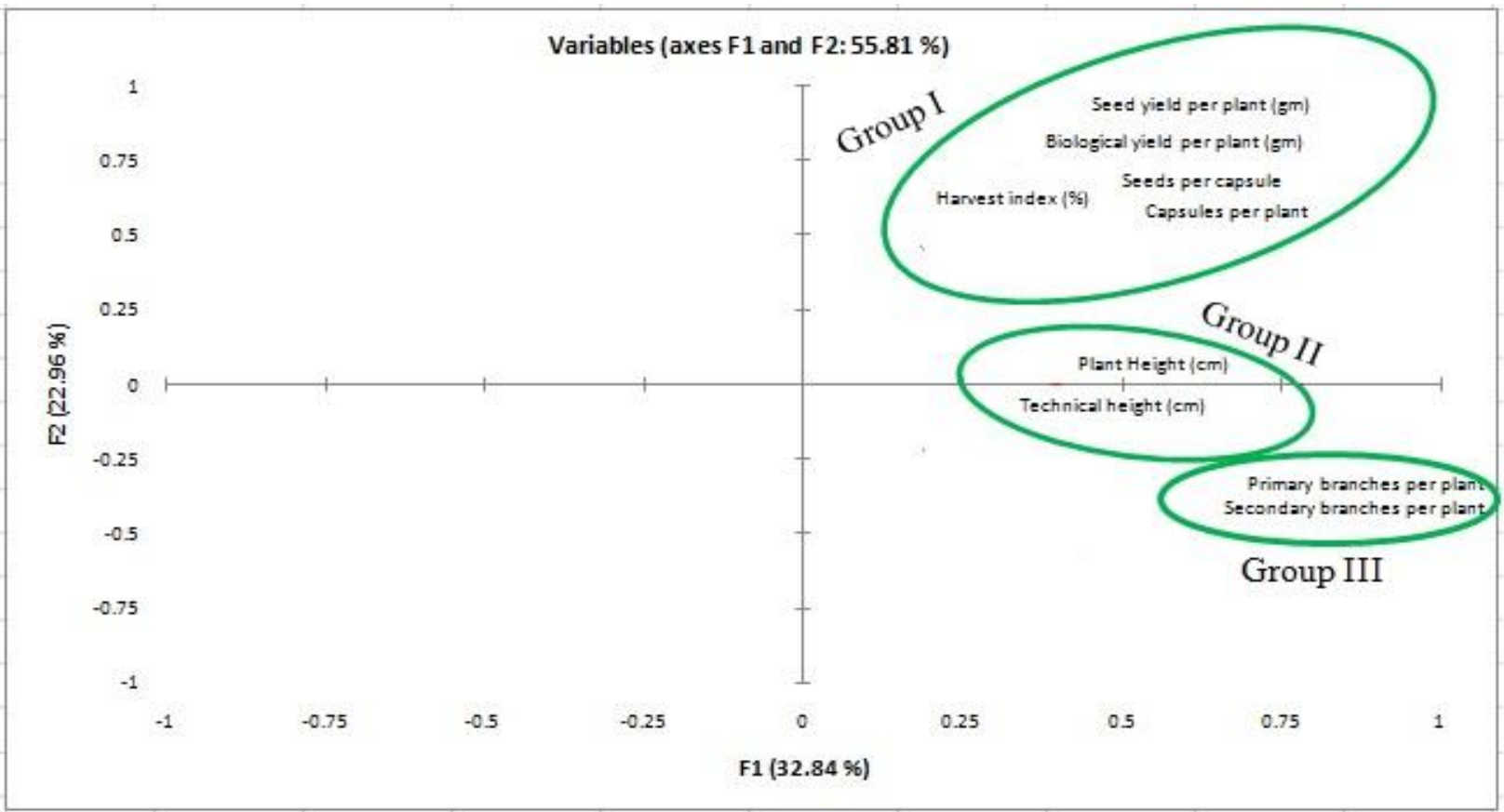


Path coefficient analysis permits the separation of the correlation coefficients into components of direct and indirect effects. Such information may be useful in predicting correlated responses of different characters towards directional selection. Keeping seed yield per plant as resultant variable and other traits as causal variables, the following results were obtained. The direct and indirect effects of genotypic path coefficients were mostly higher in magnitude than the corresponding phenotypic path coefficients (Table 3). Similar finding with respect to path coefficients have been reported by Gauraha and Rao (2011) and Reddy et al., (2013). Harvest index exerted maximum direct effect on seed yield per plant followed by biological yield per plant and seeds per capsule while plant height and primary branches per plant showed negative direct effects on seed yield per plant at both (genotypic and phenotypic) levels. Also we see that harvest index exerts maximum indirect effect via plant height followed by technical height on seed yield per plant and biological yield per plant has maximum indirect effects via capsules per plant on seed yield per plant. Seed yield had maximum genotypic and phenotypic correlation with biological yield per plant followed by harvest index and seeds per capsule so direct selection of plants based on these three traits i.e., biological yield per plant, harvest index and seeds per capsule would be effective to increase seed yield.

As path coefficient analysis determines the effect of individual traits on overall yield, principal component was also performed to determine the performance of individual advance lines and their effect on different variables.

The principal component analysis (PCA) was performed for traits (Table 4) which revealed four most informative principal components with eigen values of 2.956, 2.067, 1.674 and
0.978 respectively, which together accounted $85.273 \%$ of the total variance (Fig. 1.) for all the traits viz., plant height, technical height, primary branches per plant, secondary branches per plant, capsules per plant, biological yield per plant, seeds per capsule, seed yield per plant and harvest index. As earlier reported by Kumar and Paul (2016) The three groups were found for the traits studied (Fig. 2). The group I had 5 traits in a group (seed yield per plant, biological yield per plant, harvest index, seeds per capsule and capsules per plant) and group II had 2 traits (plant height and technical height) and in group III (primary branches per plant and secondary branches per plant) had 2 traits.

The figure shows that traits within the group are closely associated like seed yield per plant, biological yield per plant, harvest index, seeds per capsule and capsules per plant all fall under the same group it shows the traits within the group are more associated. The results showed that group I traits could be used for selecting high yielding lines. In the present investigation it is observed that there were positive significant associations between seed yield per plant with plant height, secondary branches per plant, capsules per plant, biological yield per plant, seeds per capsule and harvest index. The positive associations of such traits indicated especially that such characters can be applied for direct and indirect selection in breeding programmes. Additionally, the PCA showed that the traits fall in three groups. Group I had 5 traits including seed yield per plant so group I traits could be used for selecting high yielding lines.

\section{Acknowledgement}

The author thanks the Project Co-ordinator (Linseed) ICAR Govt. of India for financial assistance in the form of the project. 


\section{References}

Adams, M.W. 1995. An estimate of homogeneity in crop plants with special reference to genetic vulnerability in dry season, Phseolus vulgaris. Euphytica, 26: 665-679.

Akbar, M, Khan, N.I. and Sabir, K.M. 2001. Correlation and Path Coefficient Studies In Linseed. J. Biol. Sci., 1: 446-447.

Al-Jibouri, H.A., Miller, P.A. and Robinson, H.P. $\quad 1958$ Genotypic and environmental variance and covariance in upland cotton cross of inter-specific origin. Agron. J., 50: 633-36.

Dewey, D. and Lu, K.H. 1959. A correlation and path-coefficient analysis of components of crested heat grass seed production. Agron. J., 51: 515-518.

Gauraha, D. and Rao, S.S. 2011. Association Analysis for Yield and its Characters in Linseed (Linum usitatissimum L.). Res. J. Agricult. Sci., 2(2): 258-260.

Joshi, P.K. 2004. Breeding behaviour and association analysis for yield and yield component in linseed (Linum usitatissimum L.). Ph.D. Thesis, Indira Gandhi Krishi Vishwavidyalaya, Raipur, C.G.

Khan, M.L., Sharif, M., Sarwar, M., Sameea, and Ameen, M. 2010. Chemical composition of different varieties of linseed. Pak. Vet. J., 30(2): 79-82.

Kumar, N. and Paul, S. 2016. Selection criteria of linseed genotypes for seed yield traits through correlation, path coefficient and principal component analysis. J. Ani. Pl. Sci., 26(6): 16881695.

Mohammadi, S.A. and Prasanna, B.M. 2003. Analysis of genetic diversity in crop plants-salient statistical tools and considerations. Crop Sci., 43: 12351248.

Muhammad, A., Tariq, M., Anwar, M., Muhammad A., Muhammad, S. and Jafar, S. 2003. Linseed improvement through genetic variability, correlation and path coefficient analysis. Int. J. Agric. Biol., 5: 303-305.

Reddy, M.P., Arsul, B.T., Shaik, N.R. and Maheshwari, J.J. 2013. Estimation of heterosis for some traits in linseed (Linum usitatissimum L.). J. Agri and Vet. Sci., 2: 11-17.

Sohan, R., Singh, S.K. and Kerketta, V. 2004. Correlation studies in linseed (Linum usitatissimum L.). J. Res. Birsa Agri. Uni., 16: 123-126.

Tadesse, T., Singh, H. and Weyessa, B. 2009. Correlation and path coefficient analysis among seed yield traits and oil content in Ethiopian linseed germplasm. Int. J. Sustain. Crop Prod., 4: 8-16.

Tariq, M.A., Hussain, T., Ahmad, I., Saghir, M., Batool, M., Safdar, M. and Tariq, M. 2014. Association Analysis in Linseed (Linum usitatissimum L.). J. Biol. Agri. Heal., 4(6): 60-62.

Yadav, R.K. 2001. Association studies over locations in linseed. Progr. Agricult., 1(1): 11-15.

\section{How to cite this article:}

Satish Paul, Nimit Kumar and Pankaj Chopra. 2017. Improvement in Seed Yield and Related Traits of Linseed Genotypes (Linum usitatissimum L.) through Various Selection Parameters in Mid-Hills of North-West Himalayas. Int.J.Curr.Microbiol.App.Sci. 6(2): 1559-1566. doi: http://dx.doi.org/10.20546/ijcmas.2017.602.174 